\title{
Entretien avec Françoise Collin. Philosophe et intellectuelle féministe
}

Florence Rochefort et Danielle Haase-Dubosc

\section{(2) OpenEdition \\ 1 Journals}

\section{Édition électronique}

URL : https://journals.openedition.org/clio/1545

DOI : $10.4000 /$ clio. 1545

ISSN : 1777-5299

Éditeur

Belin

\section{Édition imprimée}

Date de publication : 1 avril 2001

Pagination : 195-210

ISBN : 2-85816-577-7

ISSN : 1252-7017

Référence électronique

Florence Rochefort et Danielle Haase-Dubosc, « Entretien avec Françoise Collin. Philosophe et intellectuelle féministe », Clio. Histoire, femmes et sociétés [En ligne], 13 | 2001, mis en ligne le 19 juin 2006, consulté le 23 avril 2022. URL : http://journals.openedition.org/clio/1545 ; DOI : https://doi.org/ $10.4000 /$ clio. 1545

Ce document a été généré automatiquement le 23 avril 2022.

Tous droits réservés 


\title{
Entretien avec Françoise Collin. Philosophe et intellectuelle féministe
}

\author{
Florence Rochefort et Danielle Haase-Dubosc
}

1 Françoise Collin occupe une position singulière dans le paysage intellectuel féministe depuis 1973 où elle fonda les Cahiers du GRIF. Aucun sujet touchant le « différend des sexes $»^{1}$ (selon son expression) n'a échappé, dès lors, à la curiosité de cette philosophe, écrivaine et essayiste. Après une première étape de recherches portant notamment sur Maurice Blanchot, Françoise Collin s'est consacrée prioritairement à la pensée féministe sans céder à la tentation de clore le débat par un quelconque dogmatisme et sans trahir la complexité de sa démarche philosophique.

2 Des Cahiers du GRIF mais aussi de son parcours, de son œuvre et de sa " posture » de philosophe, d'intellectuelle et de féministe, nous nous sommes entretenues avec elle le 1er février 2001 à Paris en compagnie de Danielle Haase Dubosc². L'entretien s'est poursuivi par de longues conversations et a été l'objet d'un méticuleux travail d'écriture de la part de Françoise Collin.

3 F.R : Il me semble que les Cahiers du GRIF appartiennent pleinement à l'histoire intellectuelle des années 1970-1980, par leur engagement féministe comme par les découvertes et les réflexions qu'ils ont suscitées. Comment analyses-tu aujourd'hui cette aventure d'une revue féministe?

4 F.C : Comme une aventure surprenante, à laquelle rien ne me préparait apparemment dans ma vie d'écrivaine et d'universitaire solitaire qui avait déjà trouvé ses premiers appuis éditoriaux. Une aventure à la fois individuelle et collective, intellectuelle et politique, mobilisant les forces et l'imagination pendant des années. Un long trajet de vie et de pensée.

5 Je revenais des États-Unis et j'avais découvert une vie féministe déjà très présente dans tous les domaines - et non pas confinée comme aujourd'hui dans l'institution universitaire. Ce pragmatisme américain m'avait convaincue. Nous nous épuisions en débats stériles avec le marxisme, tournant en rond entre luttes des classes et luttes de 
sexes, alors qu'elles, sans attendre, ouvraient une librairie, une galerie d'art, un centre de self help, un magazine.

6 Lors de la fondation, en 1973, des Cahiers du GRIF (Groupe de Recherches et d'Informations Féministes), qui se sont greffés sur un projet collectif - le Groupe des femmes du 11 novembre que j'avais suscité en 1972 -, nous ne pensions pas en termes de durée. Nous voulions commencer, réaliser un numéro - pour lequel nous avons rassemblé difficilement un peu d'argent. Tiré à mille exemplaires sous le titre Le féminisme pour quoi faire?, il a tout de suite été épuisé, à la librairie Maspero à Paris où il avait été déposé de même qu'à la Journée des femmes de Bruxelles. C'est ainsi que, comprenant que notre démarche correspondait à une attente, nous l'avons réédité et nous avons continué. Nous ne pensions pas d'abord à faire trace mais nous voulions faire acte. Cette attitude correspondait d'ailleurs au climat plus général de l'époque post-68 - dont je ne peux m'empêcher de garder la nostalgie : l'histoire était condensée dans l'intensité du présent : here and now.

7 Le féminisme et la fondation des Cahiers n'ont pas tant été pour moi une opération intellectuelle que la fondation d'un espace commun de rencontres, de confrontation, de pensée et d'action, un espace qui rassemblerait les femmes, non pas les seules intellectuelles mais des femmes de tous les milieux, de tous les âges, vivantes et parlantes - ma conviction ayant toujours été que la pensée pensante ne se réduit pas à ses formes théoriques et qu'elle est portée par chacun/e. La première série des Cahiers est née ainsi du souci de donner la parole à celles qui ne l'avaient pas, qui ne l'avaient jamais eue. D'où la fécondité extraordinaire de ces années dans l'ivresse des commencements. Chaque numéro était précédé de réunions et de débats avec un public partiellement neuf. Nous nous rencontrions sans nous connaître et sans présupposé, dans l'éblouissement de nous trouver confrontées à des paroles venues d'ailleurs, de les entendre, et de les trouver si proches des nôtres. Nous ne partions pas d'un espace prédéterminé : nous constituions notre espace, en parlant, en marchant, y compris dans les manifestations de rue. Nous avons ainsi identifié intuitivement les thèmes qui allaient rester les grandes articulations problématiques du féminisme: le travail, y compris domestique, la politique, le corps et la sexualité - y compris l'homosexuali-té -, le langage, la création, les violences, la maternité, ...

8 Je pense que, sans trop le savoir, quand, dans les années soixante-dix, nous avons soudain surgi sur la scène publique pour affirmer notre existence, nous avons désigné et assumé deux des enjeux majeurs de cette fin du $\mathrm{XX}^{\mathrm{e}}$ siècle et du $\mathrm{XXI}^{\mathrm{e}}$ siècle qui commence: celui de la transformation des rapports entre les sexes et de la transformation du régime de la génération et de la filiation. Même si nous n'avons pas mesuré dès le début l'ampleur du bouleversement que nous allions provoquer et/ou dont nous étions au moins le symptôme. Bien que je me défie de l'emphase, j'ose penser que nous - nous les féministes - avons été des actrices de l'histoire.

9 Après cinq ans de travail de terrain, presque sans moyens, pendant lesquels nous avions mis entre parenthèses nos trajectoires personnelles, nous nous sommes trouvées un peu usées, d'autant que nous tenions la revue à bras le corps tout en assurant des vies professionnelles et familiales. Nous avons décidé de faire une pause, d'interrompre momentanément les Cahiers. Peu après cependant deux d'entre nous, dont moi-même, avons fondé l'Université des femmes, où nous organisions des séminaires et des conférences. D'autres ont rejoint la fondation d'un magazine grand public, du nom de Voyelles. 
10 Mais l'aventure des Cahiers n'était pas épuisée et nous manquait. J'en ai, en 1981, repris l'initiative avec une partie du groupe, dont ma première co-équipière Jacqueline Aubenas et de nouvelles venues. Nous avons alors entamé la deuxième série avec une équipe partagée entre Bruxelles et Paris ou je m'étais entre-temps installée. Peu après nous avons été sollicitées par Françoise Pasquier qui a proposé de les éditer chez Tierce.

11 J'aimerais d'ailleurs rendre ici un bref hommage personnel à Françoise Pasquier, morte tout récemment, et évoquer ma collaboration durable avec elle. Elle avait d'abord été une voix inconnue me téléphonant à Bruxelles dans les années soixante-quinze, pour signaler que, travaillant pour notre office de diffusion en France et, séduite par la revue, elle avait décidé de prendre personnellement en charge sa bonne mise en place. Ainsi nous a-t-elle organisé, entre autres, une séance de débat mémorable à la librairie Autrement-dit, au coin de la rue Soufflot, en 1976, à l'occasion de la sortie du numéro Violence. Plus tard, en 1982, devenue l'éditrice des Cahiers, elle m'a permis d'ouvrir chez elle une collection - "Littérales »-, où j'ai pu publier, en plus d'Arendt, alors peu connue, Gertrude Stein mais aussi Madeleine Bourdouxhe, Yolaine Simha ou Marie Denis. Nos rapports n'étaient pas toujours simples, mais à travers l'ironie que nous partagions, la confiance, la complicité et la générosité l'emportaient. Je ne mesurais pas alors à quel point de tels moments de collaboration étroite ont été et demeurent rares et précieux.

12 Dans ces années 1980, les Cahiers se sont infléchis vers une forme un peu plus théorique, tout en restant interdisciplinaires, à mesure que la pensée féministe se fortifiait et s'affinait. À Bruxelles, d'autres membres du GRIF, dont Véronique Degraef, devaient créer plus tard - avant l'époque d'Internet - une banque de données de recherches féministes européennes, la première du genre, nommée Grace. Il y avait certainement alors un besoin général de travailler de manière plus approfondie et plus subtile, en réarticulant notre première réflexion au savoir, et d'élargir notre champ de recherche à de nouveaux thèmes, sans jamais cependant les traiter de manière purement spéculative. Pour chaque Cahier, centré sur un thème, il s'agissait toujours non pas tant de développer une thèse que d'explorer de manière plurielle un domaine. Les thèmes surgissaient de manière plus ou moins intuitive, au gré de la rencontre des questions, des circonstances ou des personnes.

13 Certains numéros étaient portés par la passion collective, d'autres par le désir d'une ou deux d'entre nous, avec la collaboration ou parfois malgré la réserve affirmée des autres. Les débats étaient parfois vifs, toujours féconds. Et chaque numéro provoquait la rencontre avec de nouvelles venues. Tous les numéros me restent très chers même s'ils sont inégalement réussis. Chacun avait sa procédure propre de constitution, était une sorte d'aventure originale. Ainsi par exemple Marie-Victoire Louis a monté seule le numéro La mise à $n u$ (1983) sur d'importantes grèves de femmes belges dans la sidérurgie (Bekaert-Cockrill), Nancy Huston, avec Leila Sebbar et Marcelle Marini, a été à l'origine du numéro littéraire Recluses/Vagabondes (1988). La dépendance amoureuse, suivi de L'Indépendance amoureuse (1985) réédité deux fois, a été notre best-seller : des hommes, dont le philosophe Jean Luc Nancy, le psychanalyste Horacio Amigorena, le théoricien d'art contemporain Thierry de Duve y avaient été associés. Nous avons d'ailleurs été plusieurs fois sur le point de constituer un comité de rédaction mixte mais il y avait des résistances farouches : l'heure de la mixité n'était pas encore venue. Le numéro Hannah Arendt (1985), que j'avais personnellement initié est un petit 
événement puisqu'il a introduit cette philosophe sur la scène féministe internationale qui l'ignorait jusque là. C'est un point sur lequel en tout cas nous avons été pionnières. La rencontre de cette philosophe - un livre sorti d'un rayon d'une librairie américaine en 1983 - a été pour moi déterminante. Bien que non féministe, elle m'a aidée à penser des problèmes internes à mon expérience féministe, à les nouer avec l'ensemble de ma réflexion politique et philosophique. C'est ainsi que j'ai poursuivi avec elle un long chemin, ponctué par des colloques, séminaires, articles, jusqu'à mon livre récent ${ }^{3}$.

Provenances de la pensée a ensuite rassemblé des contributions de femmes philosophes qui n'étaient pas non plus nécessairement féministes, contribuant à assurer l'élargissement de la notion de féminisme. Ingeborg Bachman (1987) est dû à l'initiative d'Anne-Sophie Astrup (et m'a permis la rencontre de Françoise Rétif). L'Africaine (1985) a réuni des anthropologues et ethnologues, telles que Françoise Héritier, Nicole Sindzingre, Nicole Echard, Jeanne Françoise Vincent... après avoir fait l'objet d'un colloque du GRIF à l'université de Bruxelles. Le genre de l'histoire (1988) a révélé trois jeunes chercheuses : Michèle Riot-Sarcey, Eleni Varikas, Christine Planté. De la parenté à l'eugénisme (1987), avec la collaboration de Françoise Laborie, Hélène Rouch, Marie-Jo Dhavernas, Rosi Braidotti, alertait sur les nouvelles techniques de procréation. Mais je ne peux citer ici les noms de toutes celles qui ont compté.

Un tel itinéraire n'a pas été sans conflits, intellectuels ou passionnels, mais le souci d'avancer ensemble l'emportait. On ne peut oublier que si le monde établi fonctionne avec ses règles, son ordre, ses institutions, ses places, nous avions quant à nous à inventer les formes de nos rapports, les règles d'une sociabilité nouvelle non balisée par l'institution. La question du lien entre femmes, ou féministes, reste d'ailleurs à mon avis encore à penser.

Interrompus une nouvelle fois par la fermeture assez brusque des éditions Tierce, Les Cahiers ont cependant repris plus tard leur parution chez Descartes et Cie. Le numéro consacré à Sarah Kofman, issu d'un colloque que j'avais organisé avec Françoise Proust et auquel Jacques Derrida avait participé - a été traduit en plusieurs langues. Âmes fortes, esprits libres confronte la question de l'individu et de la personne à travers des figures mystiques : il a paru à peu près au moment où Clio Histoire Femmes et Sociétés consacrait un numéro à la religion ${ }^{4}$.

17 Mais le changement d'époque, l'engagement accru des femmes dans leurs recherches individuelles et leurs carrières respectives, l'absorption quasi générale du féminisme par l'institution politique ou universitaire laissant peu de temps à des rencontres informelles, un certain assèchement aussi de la vie collective qui ne concerne pas seulement les femmes mais la société tout entière, et de nouveaux problèmes éditoriaux, ont paralysé la parution des Cahiers. Un numéro est cependant en cours de préparation, qui sous le titre Genre et justice, rassemblera des textes traduits de philosophes américaines, en débat plus ou moins serré avec John Rawls.

Ce changement de conjoncture m'a " autorisée " en quelque sorte à revenir à l'approfondissement et au rassemblement de mes propres travaux : l'énergie n'est pas infinie et on ne peut mener valablement en même temps cet approfondissement personnel de la pensée et la responsabilité d'une revue dépourvue d'infrastructure. Chaque époque a ses formes d'expression privilégiées. Et à chaque âge de la vie correspond une forme de réalisation et d'engagement à laquelle il faut se plier, tout en guettant l'émergence de nouvelles venues et de nouvelles formes d'intervention qui me 
semblent commencer à se manifester dans l'espace public. Car une époque ne se caractérise pas seulement par ses idées, mais par ses modes d'expression.

F.R. : Il semble bien que le féminisme ait permis de découvrir la valeur heuristique d'une lecture sexuée du monde et des savoirs. Comment s'est traduit ce tournant intellectuel du féminisme dans tes propres recherches et quelles thématiques ont plus particulièrement retenu ton attention?

F.C. : Mon engagement féministe des années soixante-dix m'est d'abord apparu comme une rupture radicale avec mon travail antérieur, et ce n'est que bien plus tard que j'ai pu rétablir les liens. Il m’a conduite à repérer, à réfléchir et à articuler les différents points de la structure de domination sexuée. Le fait d'animer une revue polyvalente qui n'était pas liée à un problème ou à une discipline déterminée m'a amenée à m'intéresser à de nombreux aspects directs ou indirects de cette question, que ce soit par ma curiosité personnelle ou par la rencontre de femmes, parfois d'hommes, engagé(e)s dans des recherches diverses dont je guettais l'enjeu spécifique et l'originalité pour les mettre en valeur.

21 Cette polyvalence, que je n'ai pas vécue comme un éparpillement mais comme un approfondissement, trouvait sa justification dans un rapport à la philosophie défini davantage comme une capacité de penser et de s'interroger (« rien de ce qui est humain ne m'est étranger ": Montaigne) que comme l'élaboration d'une théorie ou d'un système. J'avais été marquée d'emblée par la philosophie contemporaine - la lignée phénoménologique : Husserl, Heidegger, dont est issu le courant philosophique français de l'après-guerre, de Sartre et Merleau-Ponty à Deleuze ou Derrida en passant par Levinas. J'ai très tôt défini la politique telle que je la rencontrais dans le féminisme comme une politique plurielle et une politique de l'irreprésentable - c'est à dire sans représentation d'un " modèle »-, ce qui me confrontait à ce qu'on nomme maintenant le post-moderne avec lequel j'entretiens un rapport complexe.

22 Mais mes travaux se sont peu à peu concentrés sur certaines thématiques privilégiées, dont je retrouve la trace en compulsant mes articles (en vue d'un recueil à paraitre conjointement aux Presses Universitaire du Québec et à Paris). Ainsi ai-je été particulièrement sollicitée par la question du lien, du lien entre femmes que mettait en œuvre le féminisme, du lien social en général, et corrélativement du statut du sujet - et de son excédent par rapport à ses composantes sexuées ou autres. Dans la foulée, j’ai été amenée à m'interroger sur l'articulation privé/public, et sur les ambitions et les limites de la démocratie représentative.

La question du lien concerne à la fois le rapport synchronique des contemporains et le rapport diachronique des générations : " pluralité et natalité » était d'ailleurs le titre initialement donné à mon livre sur Hannah Arendt. Dans aucun de ces deux registres, le lien ne peut s'épuiser dans la question du contrat et la liberté dans celle de l'autonomie. Et je pense que le bouleversement du lien générationnel est un problème majeur de ce $\mathrm{XXI}^{\mathrm{e}}$ siècle. C'est un impensé de la démocratie.

Ma réflexion en rapport avec le politique n'a pas recouvert l'analyse que j'avais initialement développée autour de la littérature et de l'art, à travers mon livre sur Blanchot, issu de ma thèse de doctorat. L'articulation complexe entre poétique et politique est même peut-être le support permanent de ma réflexion. S'il y a tension entre ces deux dimensions de l'expérience humaine, il y a aussi rapport dans la mesure où il ne peut $\mathrm{y}$ avoir bouleversement politique (dans les rapports de sexe) sans bouleversement symbolique, lequel n'est pas réductible à la ponctualité de lois ou de 
mesures sociales. Le changement se cherche mais n'obéit pas au commandement. C'est dans ma réflexion sur l'écriture que j'ai appris ce que je continue à nommer « aller en direction de l'inconnu».

Parallèlement à cette réflexion, mon rapport prioritaire à la philosophie m'a portée très tôt à m'interroger sur les horizons théoriques voire idéologiques dont relevaient les courants de pensée féministe. J'ai aussi repéré la manière dont les philosophes abordent la question des femmes et de la différence des sexes. Je l'ai esquissé dans quelques articles, commencé à l'articuler et à le développer dans Le différend des sexes avant de poursuivre une enquête plus systématique dans De Platon à Derrida avec la collaboration d'Evelyne Pisier et d'Eleni Varikas ${ }^{5}$. Ce serait là mon apport de " spécialiste " au domaine du savoir. Mais j'ai aussi tenté d'interroger le rapport des femmes philosophes à leur discipline, non seulement dans le cas d'Arendt, mais dans le cas de mes contemporaines (Provenances de la pensée, Sarah Kofman).

F.R. : L'histoire, qui est l'espace de rencontre propre à la revue Clio Histoire Femmes et Sociétés, semble tenir peu de place dans ta démarche?

F.C.: Je suis bien entendu convaincue par l'importance des recherches historiques. S'agissant des femmes, il faut procéder à un véritable travail de réhabilitation qui exige sans doute la restructuration de la notion même d'histoire. Cette réhabilitation ne consiste d'ailleurs pas seulement en un relevé de marques mais aussi de traces, plus difficiles à identifier et nécessitant d'autres procédures (J'ai d'ailleurs écrit un texte intitulé « La marque et la trace $\left.»^{6}\right)$. Si j'ai accepté cet entretien, c'est précisément parce que je suis sensible à la nécessité de la mémoire et de la constitution d'une tradition, d'un matrimoine. Je pense d'ailleurs que les recherches historiques et la réflexion sur le statut même de l'histoire constituent le point fort des études féministes en France et L'histoire des femmes de Michelle Perrot leur a fait franchir le cercle des seules spécialistes.

Il est vrai que j'ai personnellement, outre mes graves lacunes, un rapport complexe à l'histoire et cela pour diverses raisons. Raisons en quelque sorte " professionnelles », la philosophie étant plutôt liée à la structure humaine qu'à son historicité, même si elle est indissociable de son origine grecque. Raisons sociologiques aussi : j'ai grandi dans un pays bilingue et binational - la Belgique - dont l'histoire étatique est courte (1830) et traversée par des enjeux divers et contradictoires: occupations tour à tour espagnole, autrichienne, française, hollandaise, qui contraignent à un certain empirisme. La résolution des problèmes présents et l'invention de l'avenir peuvent difficilement s'y appuyer sur la tradition et sur le motif de la fondation - la Révolution française - qui anime encore aujourd'hui la conscience en France. C'est le propre et la force de la culture française, me semble-t-il, que de chercher toujours dans le passé l'éclairage des enjeux présents - leur généalogie -, c'est sa richesse, et c'est peut-être aussi aujourd'hui, dans ce moment de restructuration des peuples et des états, sa difficulté. Comment répondre à la fois à la mémoire et à l'imagination?

Mais il y aussi dans mon rapport à l'histoire des composantes sans doute plus subjectives et plus obscures : mes premiers livres - des romans publiés au Seuil subvertissent spontanément l'intrigue linéaire au profit d'une temporalité interruptive, anarchique en quelque sorte, que j'aurai voulu mener jusqu'au chaos (et qui est sous d'autres formes une caractéristique du " Nouveau Roman »). Le sens émerge toujours d'un non-sens qui le borde de la contiguité plutôt que de la continuité. 

résistance il y a - porte surtout sur une conception de son développement linéaire et dialectique, dans la perspective de son accomplissement (" la Société juste », « la fin de l'histoire »).

31 F.R. : Dans ton parcours, tu ne sembles pas vouloir t'affirmer comme intellectuelle sur un seul registre, celui de la philosophe, de l'écrivaine, de la féministe, mais davantage nous entraîner sur le cheminement de ta pensée. Le cheminement? Est-ce ce qui caractérise ta démarche intellectuelle?

Oui, le terme de cheminement me convient bien, cheminement fait à la fois de continuité et de déplacements, et livré à un certain polymorphisme. Identité, mais identité composite dont certaines facettes ont prévalu au gré des circonstances. Ce cheminement est d'ailleurs celui d'une pensée qui ne s'immobilise pas dans une théorie définie une fois pour toutes à laquelle la suite ne ferait qu'ajouter des compléments ; mais qui est appelée à s'interroger et à se restructurer sans cesse. Je ne me suis jamais sentie obligée de choisir un " camp » théorique : la pensée et l'agir ne se pensent pas en ces termes. Quand des choix conjoncturels se dessinent, il faut cependant porter un jugement, prendre une décision, s'engager. Ainsi, si j'ai soutenu la parité, ce n'est pas que je la jugeais valable en termes " ontologiques »- et il me semble que Geneviève Fraisse et moi-même étions d'accord sur ce point - mais en termes stratégiques, comme une des rares propositions faites par la scène politique française du moment, une manière de la débloquer et de visibiliser a contrario la réalité de la fracture produite par la démocratie. Il nous appartient ensuite de confronter les élues à l'articulation femmes/féminisme qui ne va pas de soi. La vie politique nécessite à la fois des options hasardeuses et une vigilance sans cesse renouvelée. Il n'y a pas d'options garanties et sans effets en retour.

F.C.: La théorie constitue un cadre fixe, auquel se tenir pour évaluer les questions nouvelles. Elle n'est pas liée au moment de l'interrogation mais de l'affirmation : elle détermine les contours d'une position, voire même isole des causes. Elle séduit par sa clarté rassurante : voilà la « vérité ». Mais elle risque toujours de tourner à l'idéologie.

La pensée interroge, suit son chemin, et ses " chemins de traverse », qui " ne mènent nulle part ", pour reprendre l'expression de Heidegger, mais qui ouvrent des clairières. La pensée retient la question dans son affirmation même. Elle prétend éclairer mais non déterminer la décision. Elle nourrit et fracture tout à la fois la théorie.

La théorie fait thèse : elle est identifiable et communicable comme un objet. Une amie américaine m'a fait remarquer que dans mes articles la thèse n'est pas énoncée assez clairement (comme elle doit l'être en début de texte pour faciliter la lecture, semble-til). Mais il me semble toujours que la «thèse » doit se découvrir à la lecture, non dans un résumé, qu'elle est inséparable du texte. Et j'attends même de la lecture qu'elle découvre dans le texte ce que je ne sais pas nécessairement y avoir mis : c'est cela le travail de l'interprétation qui déplie ce qui était plié.

37 Je conçois la théorie comme un échafaudage, un bricolage transitoire, un pont de fortune qui assure les passages, non comme un édifice où demeurer. Ou comme une sorte de boite à outils.

38 Le langage est toujours menacé par l'encodage idéologique, auquel risque de succomber la théorie. Elle doit être constamment rouverte, et la conquête des certitudes ravivée 
par l'incertain : le mouvement, et le mouvement des femmes, si bien nommé, est un « aller vers ». La théorie commande. La pensée explore.

D.H.D : Tu nous as donné souvent le courage de ne pas nous laisser enfermer dans l'orthodoxie et, entre autres, dans les impasses des débats autour de la spécificité.

F.C.: J'ai été moi aussi travaillée dès le début par ce débat entre spécificité et universalité, dualisé et figé me semble-t-il pour des raisons à la fois propres à l'histoire du féminisme français et à la tradition culturelle nationale. Mais il vient un moment où le débat se transforme en alternative idéologique stérile. Il doit être déplacé, contourné, revivifié pour ne pas bloquer la pensée dans une "logique des contraires ». Le véritable abandon de tout postulat métaphysique (ou naturaliste) des sexes, c'est le dépassement de la position unitaire autant que duelle. J'ai parlé de " l'irreprésentable » mais aussi de la « praxis » des sexes. Les questions doivent toujours être portées, elles ne doivent pas nous mettre à la question sous la forme d'une alternative.

Le féminisme n'est en tout cas pas pour moi un « devenir homme » des femmes, au sens d'une conversion au monde constitué, marqué du règne de l'avoir, au sens d'une égalité pensée en terme d'égalisation à ce qui est, à ceux qui sont, mais un travail de réinvention des positions sexuées et du monde commun. Je reviendrais sur ce point à l'analyse hégéliano-marxiste: c'est l'opprimé qui est porteur de valeurs nouvelles, voire universelles, non en raison de sa " nature ", bonne ou supérieure, mais de sa position historique. "Femmes » continue pour moi à faire sens - un sens ouvert - et je me méfie d'un effacement prématuré de la frontière des sexes, d'une "indifférence " supposée qui se ferait au profit de la position dominante.

D.H.D : Ce qui est frappant dans tes textes, en particulier dans ton livre Je partirais d'un mot Le Champ symbolique, c'est ta pudeur, ta retenue. Plus qu'une convention ou une habitude de l'enfance, cela me semble faire partie de la démarche de ta pensée. Les zones non dites permettent à ce qui est dit d'avoir une force toute particulière.

F.C. : La pensée formulée émerge toujours d'un fond de non dit. La pensée pensée est comme l'écume d'une pensée pensante qui n'a pas trouvé ses mots. C'est pourquoi il faut toujours recommencer à dire, à redire : on n'en a jamais fini. Achever un texte, un livre c'est accepter de renoncer à tout ce qu'il fallait dire et dont une faible partie a émergé.

Il me semble d'ailleurs qu'il y a souvent chez une femme ou dans une œuvre de femme plus que ce qu'elle énonce ou formule, qu'il y a un au-delà, un reste par rapport à son énoncé, alors qu'un homme joue son va-tout dans son énoncé, s'y épuise en quelque sorte, épuise sa subjectivité dans l'objectivation. Aussi, pour moi, commencer à écrire est toujours heureux, mais finir, accepter de n'avoir réussi à dire que ce peu, est toujours difficile. La fin, qui devrait être un soulagement et même une satisfaction, m'apparaît surtout dans ce qu'elle a manqué. Aussi rencontrer une lectrice, un lecteur qui a lu dans le texte ce qui y est sans y être explicité est un bonheur.

D.H.D. : Je t'ai toujours considérée comme une intellectuelle de la liberté. En échappant à la théorie, la pensée ne devient-elle pas innovatrice?

F.C. : La théorie comporte en effet un élément de fixité, d'ailleurs rassurante. Elle vire facilement à l'idéologie. Ma réserve vient sans doute de la confusion entre théorie et idéologie que j'ai affrontée dans mon époque marxiste et dont j'ai toujours voulu me garder dans le féminisme. Au " ou bien, ou bien " je préfère le " ni, ni ». Ce qui m'intéresse c'est, dans ce qui a été dit, ce qui est encore à dire, le reste, ce qui n'est pas 
encore advenu en quelque sorte : ce qui est resté en friche. Penser c'est sortir du cercle qu'on a déjà tracé, en repérer la limite pour la franchir. Je suis toujours sensible à ce qui fait problème dans un énoncé, que ce soit celui des autres ou le mien, ce qui dérange et force à continuer à penser. Ainsi, dans la relecture des philosophes que j'ai effectuée récemment, sur le fil de la question des femmes, c'est pour chacun son débat interne, voire ses contradictions, qui m'intéressent, ce qu'il ferme péremptoirement et ce qu'il laisse ouvert.

47 J'aime le nouveau, ou ce qui m'apparaît comme nouveau car bien souvent je reviens sans le savoir à ce que j'avais déjà traversé mais par une voie différente. Un parcours, c'est à la fois un déplacement permanent et la persistance de schèmes récurrents. C'est sans doute ce que Nietzsche désigne dans le phénomène de la répétition qui commande le déplacement.

D.H.D. : C'est ton côté surréaliste : le hasard objectif décide; c'est à partir de là que l'émotion suscite la pensée...

49 F.C. : L'image que tu me renvoies ainsi me confirme que je ne suis pas une théoricienne opérant dans la continuité d'une construction mais que j'ai souvent fonctionné par à coups, sensible à la rencontre, à la requête des circonstances et des personnes, voire des paysages, au coup de cœur en quelque sorte, à l'intuition. Pourtant, en même temps, j'ai le sentiment que dans ce jeu du hasard se manifeste une unité fondamentale, voire parfois une certaine fixité : souvent, relisant mes textes d'il y a vingt cinq ans, il me semble qu'y était déjà dit ce que j'ai cru ensuite découvrir à grand peine. L'avantage des trous de mémoires, c'est d'éprouver toujours le nouveau.

Mais on peut aussi interpréter cette procédure de la pensée conduite par ce que tu nommes « le hasard objectif » d'une manière différente. La forme même qu'a prise la pensée féministe comporte de nombreux éléments conjoncturels : on pense parce qu'un possible s'ouvre, parce qu'une proposition vient de l'autre ou des circonstances. On pense sur demande, ce qui ne signifie pas sur commande. La pensée surgit de la rencontre, rencontre de l'événement, des personnes, appel auquel on répond (tout en décidant toujours que c'est bien la dernière fois). C'est dans l'ordre de la pensée ce qui reste de l'aventure du féminisme comme instance plurielle. J'en vois à la fois la richesse et les limites. Richesse du dialogue explicite ou anonyme constitutif d'une scène commune toujours mouvante; limites contraignantes de cette scène même qui rend difficile sa transgression, le libre cours d'une pensée impertinente ou "gratuite ». L'interpellation surprend mais retient en quelque sorte dans le cercle.

51 F.R. : La notion d'intellectuelle que nous souhaitons explorer dans ce numéro (et qui s'enracine dans une réflexion sur la longue durée - de l'Antiquité à nos jours -) nous semble devoir permettre d'échapper aux clivages entre l'écrivaine, la philosophe et la féministe engagée. Il s'agirait à la fois une posture d'écriture et d'un rapport critique au monde. Qu'en pense- tu ?

52 F.C. : Oui, être une intellectuelle, en ce sens là, c'est se tenir entre la pensée et l'action, entre le retrait et l'exposition à la scène publique, entre l'élaboration solitaire de la pensée et de l'écriture et l'affrontement des enjeux collectifs, entre l'intemporel et le temps donné, ici et maintenant, celui auquel, sans que nous l'ayons choisi, nous avons à faire. C'est répondre à l'interpellation du donné et en même temps l'excéder, en être libre. 

quelques exceptions près, celles de l'intellectuel homme. Celui-ci est en quelque sorte une "fonction " reconnue de la société donnée, voire sa fonction critique convenue. Certains dominent la scène et, relayés par les media, sont les consultants récurrents des grands problèmes du jour; d'autres sont constitués de la même manière comme les critiques ou les contestataires professionnels, dont les noms figurent au bas des pétitions publiées par les journaux et qui sont au premier rang du rituel des manifestations. La société actuelle est assez intégrée pour que l'intellectuel, même dans la critique, y soit une figure " autorisée » qui y a sa place et est à sa place. Il n'y a plus de figure interruptive. L'interruption semble n'avoir plus de recours que dans la violence. lintellectualité éclairée. Notre intellectualité critique de femmes reste encore très largement officieuse. Peu d'intellectuelles sont convoquées aux débats publics (sauf éventuellement s'il s'agit de question de femmes). Mais peu aussi, il est vrai, incarnent encore aujourd'hui l'anarchie. Le féminisme est majoritairement passé de l'insurrection à l'institution, avec des gains certains, et avec des pertes. J'avais autrefois défini le féminisme comme résistance, voire mouvement de guérilla : est-ce encore soutenable? Il faut en tout cas garder rapport avec cette origine, préserver même dans l'institution l'instance critique. Blanchot, le rapport à l'inconnu qu'engage l'une et l'autre dimension. Dans le langage comme dans l'agir, il s'agit d'aller vers ce qui n'est pas encore, de faire être du nouveau, même si ce n'est pas la révolution, mais un déplacement. certaine lecture des enjeux d'aujourd'hui?

F.C. : Oui, c'est pour moi une grille de lecture prioritaire, mais si intériorisée que je ne dois plus y recourir explicitement : elle m'habite. J'hésiterais donc à parler de grille de lecture, comme si je ne lisais le réel qu'à travers elle. C'est devenu plutôt une manière d'être au monde: une grille ouverte, qui après m'avoir peut-être conditionnée temporairement me rend libre, un questionnement qui croise les autres.

61 J'ai toujours voulu laisser ré-interroger la pensée par la pratique et accompagner la pratique par la vigilance de la pensée. Je recours volontiers au concept de praxis, rencontré dans le marxisme, mais présent aussi chez Arendt dans sa référence à 
Aristote, pour désigner à la fois la pensée et la pratique. Arendt pense que le politique ne peut être commandé par une philosophie préalable qui lui fournirait sa norme, mais que le politique comme agir suscite constamment son propre objet. L'imagination et la faculté de juger sont pour elles les qualités essentielles du politique. La pensée est une vigilance qui accompagne et qui éclaire, mais non une théorie qui détermine l'action à partir d'une représentation préalable des objectifs auxquels le réel aurait à se plier. L'essence $d u$ politique consiste dans l'élaboration et la mise en forme du terrain d'action, non dans le choix entre deux objets prédéterminés et dans l'injonction du " pour ou contre ». Il est vrai que le caractère passionnant du féminisme était, et reste pour moi, lié à ce projet premier : dessiner le paysage de la liberté plutôt que d'en définir les objets. L'insurrection ou si on préfère la déconstruction plutôt que l'institution.

Il y a cependant une crainte que je ne peux m'empêcher de formuler: l'enjeu du féminisme a été et est si important et si absorbant qu'il occulte souvent les paramètres socio-économiques et culturels qui le traversent et le rend trop souvent imperméable aux autres enjeux de notre temps. Il produit parfois un effet de bulle, bulle dans laquelle semblent ne pas pénétrer les tragédies du post-colonialisme, du racisme, des guerres, des génocides contemporains ou tout simplement le polymorphisme de la société française. La politisation radicale d'une dimension prioritaire entraîne une dépolitisation qui pourrait se confondre avec une indifférence pouvant devenir criminelle. Pour comprendre cette remarque, il faut se souvenir, comme l'a fait Rita Thalmann, de ce qu'il en a été du féminisme et des féministes au moment du nazisme et de l'antisémitisme. Il y a là un risque permanent. Les débats qui ne touchent pas directement à la question du genre restent étrangement absents de la scène féministe (même si certaines s'engagent à titre individuel en dehors d'elle): est-ce par indifférence ou pour éviter des conflits internes au mouvement? Le féminisme est un vecteur prodigieusement fécond de lecture du monde, mais ce n'est cependant qu'un vecteur. Il me semble que j'ai écrit quelque part : il faut être féministe, mais il faut être plus que féministe : je m'en tiendrais momentanément là.

D.H.D. : Tu attaches beaucoup d'importance à la portée symbolique du féminisme. La définition de Paul Valéry que cite le Petit Robert : « Le métier des intellectuels est de remuer toutes choses sous leurs signes, noms ou symboles » semble te convenir.

F.C.: C'est une définition très intéressante. Elle tranche avec celle qui est habituellement reçue et qui lie davantage l'intellectuel à son intervention publique. Cette définition touche aux couches profondes du politique, reportant l'attention sur l'importance du tissu même de l'être ensemble, travaillé par la pensée, le langage, la création, sur tout ce qui dans cet être ensemble n'est pas suspendu à la visibilité d'un décret, d'une décision politique au sens ponctuel du terme. Dans une telle optique, les effets du travail de l'intellectuel/le ne sont pas immédiatement mesurables ni calculables.

Il/elle est assigné à une tâche qui est de déplacement plutôt que de bouleversement ponctuel, tâche qu'il lui faut assumer singulièrement, dans un espace donné, qui est le sien, et non par un surplomb totalisant. J'aurais jusqu'ici défini de cette manière plutôt l'écrivain ou l'artiste, en proie aux signes, mais comment ne pas y joindre en effet celui/celle qui pense, déplace les angles de vue, découvre des paysages inédits. Nous voyons bien, avec le recul, que nous avons produit des effets souterrains qui s'étendent en dehors même de notre maîtrise et du mesurable. Car le changement des signes et des 
symboles ne relève pas d'une décision brusque mais d'un lent travail souterrain. Une telle définition rend son importance à chaque démarche singulière, solitaire souvent, parfois déprimée par son apparente impuissance. Nous ne savons pas ce qui dans notre démarche est stérile ou fécond à terme : nous marchons, tant que nous pouvons. Chaque pensée, chaque mot prononcé avec rigueur compte pour le destin du monde commun.

\section{BIBLIOGRAPHIE}

Collin Françoise,

--, 1960, Le jour fabuleux, Paris, Seuil.

-, 1961, Rose qui peut, Paris, Seuil.

-, 1971, Maurice Blanchot et la question de l'écriture, Paris, Gallimard, rééd. coll. Tel, 1988.

-, 1975, 331 W 20, Lection du président, Bruxelles, Transédition.

-, 1988, Le rendez-vous, Paris, Tierce.

-, 1988, Le jardin de Louise, Montréal, NBJ.

_, (dir.), 1992, Le sexe des sciences, Paris, Autrement.

-, 1999a, Hannah Arendt, Paris, Odile Jacob.

-,1999b, Le différend des sexes De Platon à la parité, Nantes, Éditions Pleins Feux.

-, 1999c, Je partirais d'un mot Le Champ symbolique, Paris, Fus Art.

-, avec Pisier Evelyne et Varikas Eleni, 2000, Les femmes de Platon à Derrida. Anthologie critique, Paris, Plon.

\section{NOTES}

1.Collin 1999b.

2.Danielle Haase-Dubosc, spécialiste du xvire siècle et auteure dans ce numéro, et a été membre du comité de rédaction des cahiers du GRIF.

3.Collin 1999a, en voir le compte-rendu de Diane Lamoureux dans la rubrique « Clio a lu » de ce numéro.

4. Clio Histoire, Femmes et sociétés « Femmes et religions » 2/1995 numéro coordonné par Agnès Fine et Claudine Leduc.

5.Collin Pisier Varikas 2000.

6.Collin 1999c : 54-63. 